\title{
EXPLICIT KREIN RESOLVENT IDENTITIES FOR SINGULAR STURM-LIOUVILLE OPERATORS WITH APPLICATIONS TO BESSEL OPERATORS
}

\author{
S. Blake Allan, Justin Hanbin Kim, Gregory Michajlyszyn, \\ ROGER NiCHOLS AND DON RUNG
}

\begin{abstract}
We derive explicit Krein resolvent identities for generally singular Sturm-Liouville operators in terms of boundary condition bases and the Lagrange bracket. As an application of the resolvent identities obtained, we compute the trace of the resolvent difference of a pair of self-adjoint realizations of the Bessel expression $-d^{2} / d x^{2}+\left(v^{2}-(1 / 4)\right) x^{-2}$ on $(0, \infty)$ for values of the parameter $v \in[0,1)$ and use the resulting trace formula to explicitly determine the spectral shift function for the pair.
\end{abstract}

Mathematics subject classification (2010): 47A10, 47A55, 47A56, 47B10.

Keywords and phrases: Krein identity, singular Sturm-Liouville operator, Bessel operator, spectral shift function.

\section{REFERENCES}

[1] M. Abramowitz And I. A. Stegun, Handbook of Mathematical Functions, Dover, New York, 1972.

[2] N. I. Akhiezer and I. M. Glazman, Theory of Linear Operators in Hilbert Space, Volume II, Pitman, Boston, 1981.

[3] A. Alonso And B. Simon, The Birman-Krein-Vishik theory of selfadjoint extensions of semibounded operators, J. Operator Theory 4 (1980), 251-270.

[4] A. ANANIEVA AND V. BUdyiKa, To the spectral theory of the Bessel operator on finite interval and half-line, J. Math. Sci. 211, no. 5 (2015), 624-645.

[5] A. Yu. ANAN'EVA AND V. S. BUdYKa, On the spectral theory of the Bessel operator on a finite interval and the half-line, Diff. Eq. 52 (2016), 1517-1522.

[6] T. Ando AND K. Nishio, Positive selfadjoint extensions of positive symmetric operators, Tohoku Math. J. (2), 22 (1970), 65-75.

[7] M. Ashbaugh, F. Gesztesy, M. Mitrea, R. Shterenberg, and G. Teschl, The Krein-von Neumann extension and its connection to an abstract buckling problem, Math. Nachr. 283, No. 2 (2010), 165-179.

[8] L. Bruneau, J. Derezińs Ki, AND V. Georgescu, Homogeneous Schrödinger operators on halfline, Ann. H. Poincaré 12 (2011), 547-590.

[9] S. Clark, F. Gesztesy, R. Nichols, And M. Zinchenko, Boundary data maps and Krein's resolvent formula for Sturm-Liouville operators on a finite interval, Op. Mat. 8 (2014), 1-71.

[10] J. DEREZIŃSKI AND S. RICHARD, On Schrödinger operators with inverse square potentials on the half-line, Ann. H. Poincaré 18, 869-928 (2017).

[11] J. DEREZIŃSKI AND S. RICHARD, On radial Schrödinger operators with a Coulomb potential, Ann. H. Poincaré 19 (2018), 2869-2917.

[12] J. Eckhardt, F. GeszTesy, R. Nichols, And G. TeSChl, Weyl-Titchmarsh theory for SturmLiouville operators with distributional potentials, Opuscula Math. 33 (2013), 467-563.

[13] W. N. EveritT, A catalogue of Sturm-Liouville differential equations, In: W. O. Amrein, A. M. Hinz, D. P. Pearson (eds), Sturm-Liouville Theory. Birkhäuser, Basel, 2005. 
[14] W. N. EveritT AND H. KAlf, The Bessel differential equation and the Hankel transform, J. Comp. App. Math. 208 (2007), 3-19.

[15] F. Gesztes y, Y. Latushrin, M. Mitrea, and M. Zinchenko, Non-self-adjoint operators, infinite determinants, and some applications, Russ. J. Math. Phys. 12 (2005), 443-471.

[16] F. GesZTESy, L. LitTLEJOHN, AND R. Nichols, On self-adjoint boundary conditions for singular Sturm-Liouville operators bounded from below, in preparation.

[17] F. GesZtesy AND B. Simon, Uniqueness theorems in inverse spectral theory for one-dimensional Schrödinger operators, Trans. Amer. Math. Soc. 348 (1996), 349-373.

[18] F. Gesztesy and E. TseKanovskit, On matrix-valued Herglotz functions, Math. Nachr. 218 (2000), 61-138.

[19] K. Kirsten, P. LOYA, AND J. PARK, The very unusual properties of the resolvent, heat kernel, and zeta function for the operator $-d^{2} / d r^{2}-1 /\left(4 r^{2}\right)$, J. Math. Phys. 47, (2006), 043506, $27 \mathrm{pp}$.

[20] M. G. KREIN, The theory of self-adjoint extensions of semi-bounded Hermitian transformations and its applications. I, Mat. Sb. (N.S.) 20 (1947), 431-495 (in Russian).

[21] M. A. NAimark, Linear Partial Differential Operators Part II. Linear Differential Operators in Hilbert Space, Transl. by E. R. Dawson, Engl. translation edited by W. N. Everitt, F. Ungar Publishing, New York, 1968.

[22] M. Reed and B. Simon, Methods of Modern Mathematical Physics. II: Fourier Analysis, SelfAdjointness, Academic Press, New York, 1980.

[23] K. SchmüDgEn, Unbounded Self-Adjoint Operators on Hilbert Space, Graduate Texts in Mathematics, Springer, New York, 2012.

[24] G. Teschl, Mathematical Methods in Quantum Mechanics. With Applications to Schrödinger Operators, 2nd ed., Graduate Studies in Math., Vol. 157, Amer. Math. Soc., RI, 2014.

[25] G. N. Watson, A Treatise on the Theory of Bessel Functions, Second Edition, Cambridge University Press, 1944.

[26] J. Weidmann, Spectral Theory of Ordinary Differential Operators, Lecture Notes in Mathematics, Vol. 1258, Springer-Verlag, 1987.

[27] D. R. YAfaeV, Mathematical Scattering Theory, Transl. Math. Monographs, Vol. 105, Amer. Math. Soc., Providence, RI, 1992.

[28] A. Zettl, Sturm-Liouville Theory, Mathematical Surveys and Monographs, Vol. 121, Amer. Math. Soc., Providence, RI, 2005. 